\title{
Supporting anticoagulant treatment decision making to optimise stroke prevention in complex patients with atrial fibrillation: a cluster randomised trial
}

\author{
Melina Gattellari ${ }^{1,2 *}$, Andrew Hayen ${ }^{3}$, Dominic Y. C. Leung ${ }^{4,5}$, Nicholas A. Zwar ${ }^{6}$ and John M. Worthington ${ }^{1,4}$
}

\begin{abstract}
Background: Anticoagulation for preventing stroke in atrial fibrillation is under-utilised despite evidence supporting its use, resulting in avoidable death and disability. We aimed to evaluate an intervention to improve the uptake of anticoagulation.

Methods: We carried out a national, cluster randomised controlled trial in the Australian primary health care setting. General practitioners received an educational session, delivered via telephone by a medical peer and provided information about their patients selected either because they were not receiving anticoagulation or for whom anticoagulation was considered challenging. General practitioners were randomised to receive feedback from a medical specialist about the cases (expert decisional support) either before or after completing a post-test audit. The primary outcome was the proportion of patients reported as receiving oral anticoagulation. A secondary outcome assessed antithrombotic treatment as appropriate against guideline recommendations.

Results: One hundred and seventy-nine general practitioners participated in the trial, contributing information about 590 cases. At post-test, 152 general practitioners (84.9\%) completed data collection on 497 cases (84.2\%). A 4.6\% (Adjusted Relative Risk $=1.11,95 \% \mathrm{Cl}=0.86-1.43$ ) difference in the post-test utilization of anticoagulation between groups was not statistically significant $(p=0.42)$. Sixty-one percent of patients in both groups received appropriate antithrombotic management according to evidence-based guidelines at post-test (Adjusted Relative Risk $=1.0 ; 95 \% \mathrm{Cl}=0.85$ to 1.19$)(p=0.97)$.
\end{abstract}

Conclusions: Specialist feed-back in addition to an educational session did not increase the uptake of anticoagulation in patients with AF.

Trial registration: ANZCTRN12611000076976 Retrospectively registered.

Keywords: Atrial fibrillation, Knowledge translation, Stroke prevention

\footnotetext{
* Correspondence: melina.gattellari@health.nsw.gov.au

${ }^{1}$ Department of Neurology, Institute for Clinical Neurosciences, Neuroscience Research, Royal Prince Alfred Hospital, Missenden Road, Sydney Local Health District, Camperdown (Sydney), New South Wales 2050, Australia

${ }^{2}$ Ingham Institute for Applied Medical Research, 1 Campbell Street, Liverpool, New South Wales 2170, Australia

Full list of author information is available at the end of the article
}

\section{Background}

Affecting 1 in 20 people over the age of 65 and one in 10 people older than 75 years [1], atrial fibrillation (AF) is associated with a five-fold increased risk of ischaemic stroke [2]. AF-related strokes are more likely to be fatal and disabling than those occurring in patients without

C C The Author(s). 2020 Open Access This article is licensed under a Creative Commons Attribution 4.0 International License, which permits use, sharing, adaptation, distribution and reproduction in any medium or format, as long as you give appropriate credit to the original author(s) and the source, provide a link to the Creative Commons licence, and indicate if changes were made. The images or other third party material in this article are included in the article's Creative Commons licence, unless indicated otherwise in a credit line to the material. If material is not included in the article's Creative Commons licence and your intended use is not permitted by statutory regulation or exceeds the permitted use, you will need to obtain permission directly from the copyright holder. To view a copy of this licence, visit http://creativecommons.org/licenses/by/4.0/ The Creative Commons Public Domain Dedication waiver (http://creativecommons.org/publicdomain/zero/1.0/) applies to the data made available in this article, unless otherwise stated in a credit line to the data. 
AF [3], representing a significant burden for individuals, their families and the wider the community.

The long-standing evidence-practice gap in AF management is widely documented [4-10]. Evidence from randomised controlled trials confirms anticoagulation as the most effective antithrombotic to prevent stroke in AF [11]. However, at least one-half of people with AF do not receive anticoagulation and up to $85 \%$ experiencing an acute ischaemic stroke are either not anticoagulated or have sub-therapeutic anticoagulation $[5,7,8]$.

General practitioners (GPs) have considerable reservations about prescribing anticoagulants, appearing to be "more influenced by the events they induce (bleeds) than the events potentially prevented, in this case devastating strokes" [12]. However, the benefits of anticoagulation outweigh the risks for the majority of people with AF, including for those with elevated bleeding risks such as the elderly [4-10]. Only around $2 \%$ of people with AF have an absolute contraindication to oral anticoagulants [10].

Barriers to evidence-based management must be overcome to minimise stroke risk. Implementation strategies such as academic detailing and audit and feedback may increase evidence-based practices [13, 14]. Opinion led educational programs may also effect change in patient management [15]. At the time of designing the study reported here, to our knowledge, there were only two randomised evaluations of implementation strategies designed to promote anticoagulant prescribing specifically targeting GPs. Ornstein et al. [16] implemented quarterly audit and written feedback reports about the management of several conditions, including AF, randomising 10 out of 20 practices to receive educational site visits and interpractice meetings to share approaches to improve patient management. Changes in anticoagulant prescribing did not significantly differ between study arms. Audit and feedback reports presented the percentage of anticoagulated AF patients and were not focussed on individual patient cases for which tailored and specific advice may be necessary to overcome barriers to anticoagulant prescribing. Several stroke risk factors were simultaneously targeted, which may have displaced attention from managing $\mathrm{AF}$ to arguably less challenging care, such as blood pressure, glucose and lipid level monitoring, which were successfully modified. In another trial, a multifaceted intervention including an educational outreach visit increased $\mathrm{AF}$ case-finding but not appropriate antithrombotic prescribing [17]. The effect on anticoagulant prescribing was not reported. In Australia, a non-randomised study found that guideline dissemination and educational outreach targeting GPs increased warfarin use demonstrating the promise of these strategies [18].
Specialist opinions about how to best manage AF may be highly influential, guiding GP decision-making. In everyday practice there are limited opportunities for GPs to liaise with specialists for educational purposes. In one randomised controlled trial of stroke survivors, including 32 patients with AF, a model encouraging shared care between discharging specialists and GPs mediated by a nurse educator improved stroke risk management [19].

We therefore conducted a randomised controlled trial of an intervention targeting barriers to anticoagulation prescribing. We evaluated the impact of specialist communication about stroke prevention exclusively in $\mathrm{AF}$, providing GPs with the opportunity to discuss specific patient cases and barriers to management with a medical peer, followed by expert decisional support to consolidate decision making. We hypothesised that a significantly higher proportion of cases managed by GPs provided with expert decisional support would receive anticoagulation compared with cases whose GPs received academic detailing in the form of an educational session alone.

\section{Methods}

The study protocol has been published [20] and methods are summarised here. The study adheres to consort criteria for the reporting of cluster randomised controlled trials. The study was conducted between 2010 to 2012, incorporating GP recruitment commencing in 2010 and final post-test data collection in 2012.

\section{Setting}

Almost 90\% (88.6\%) of the full-time equivalent Australian GP workforce is in private practice [21] and around $85 \%$ of Australian residents consult a GP at least once a year [22]. Private practice encompasses primary care assessments, preventive medicine, the ongoing care of patients with chronic illness, care of minor acute illness, hospital referrals and access to specialist and diagnostic services. Residents can consult any GP of their choosing irrespective of residential location. Consultation costs are subsidised by Australia's universal health insurance scheme.

\section{Participant recruitment}

As described elsewhere [20], a random sample of GPs from across Australia was selected and approached by mail. GPs participating in another study conducted by the authors were not contacted. We first undertook a pilot of recruitment methods to inform the number of GPs we needed to contact to achieve the required sample size. This was followed by two phases of recruitment (2010 and 2011), during which we carried out two trials of response-aiding strategies [20], the results of which will be reported elsewhere. GPs were asked to register 
their interest in the program and those so doing were mailed further information and a consent form. Trainee GPs and GPs who indicated that they were not in active private practice or whose practice did not include elderly patients were considered ineligible.

Consenting doctors received written educational materials followed by an educational session, delivered via telephone by a medically trained peer, also a GP. The educational session was consistent with academic detailing as the session was designed to be interactive, with peers detailing the mailed educational materials with the GP participants. The GP peers reinforced appropriate practice and conveyed evidence-based information to address practice that was not consistent with recommended management. We use the term "academic detailing" to label the interactive one-to-one educational component of the program and this term is used from here on.

The content of the educational materials, reported in our protocol [20], was mirrored during the academic detailing session to reinforce messages. Briefly, medical peers discussed the epidemiology of AF and stroke outcomes of AF-related stroke, risk calculation of stroke and expected risk reduction associated with anticoagulation and antiplatelet medication. Stroke risk was calculated using the $\mathrm{CHADS}_{2}$ score (one point each for Congestive Heart Disease, Hypertension and Age over 75 and two points allocated for prior history of stroke or transient ischaemic attack) [23] with scores ranging from 0 for patients without any $\mathrm{CHADS}_{2}$ risk factors to 6 for those with all $\mathrm{CHADS}_{2}$ comorbidities. Both medical peers and GP participants had access to a table reporting baseline stroke risk per 1000 patient years for each value of the CHADS ${ }_{2}$ score based on those reported by Gage, Waterman, Shannon et al. [23]. The absolute risk with either warfarin or aspirin was presented to GPs calculated by applying an estimated benefit of a $67 \%$ relative risk reduction for warfarin and a $21 \%$ relative risk reduction for aspirin [24]. For example, a $\mathrm{CHADS}_{2}$ risk score of 3 corresponds to an absolute annual risk of stroke of $5.9 \%$, reduced by $67 \%$ and $21 \%$ in relative terms to $1.9 \%$ with warfarin and $4.7 \%$ with aspirin, respectively.

To explore barriers, GPs were asked how many out of 10 patients with AF over the age of 65 they would anticoagulant. Medical peers asked GPs what would characterise patients not receiving anticoagulation and then systematically addressed reasons offered against anticoagulation. Information predominately pertained to warfarin as the only government subsidised anticoagulant available for managing AF during the course of the study.

\section{Pre-test data collection informing primary and secondary outcomes}

During the academic detailing session, GPs provided details about their selected AF cases, including age, sex, type of AF (paroxysmal versus chronic) and stroke risk factors to enable calculation of the $\mathrm{CHADS}_{2}$ score [23]. History and current use of antithrombotic medication were also determined. Medical history relating to actual or perceived barriers to anticoagulation were reported. We advised GPs to identify 3 to 4 AF patients during the first and at least five patients during the second phase of recruitment who were not receiving anticoagulation or for whom management was challenging. Therefore, the intervention was designed to support GP decision-making where decisions to prescribe anticoagulation was perceived to be complex or uncertain thus identifying patient cases in whom barriers to anticoagulation would be encountered. Medical peers and GPs discussed relevant guideline recommendations for either anticoagulation or antiplatelet medication based on stroke risk and the American Chest Physician Guidelines current at the time the study was conducted [25]. Safety issues were addressed including combination antithrombotic treatment, effect of hypertension on stroke risk, anticoagulant use in patients with a history of intracerebral haemorrhage and regulatory authority safety alerts for direct oral anticoagulants (DOACs), where applicable. GPs were then given the opportunity to pose questions to be answered by specialists.

\section{Randomization and allocation concealment}

Academic detailers noted the date and time of the academic detailing session indicating entry into the study. GPs were ordered according to their entry into the study and randomised by a statistician independent to the research team to either receive expert decisional support or to academic detailing alone, receiving decisional support after the post-test phase was completed. Block randomization was stratified according to the number of cases per GP ( $\leq 2$ or $>3)$, using a block size of two, four or six, with block size randomly varied.

\section{Expert decisional support intervention}

Information completed during academic detailing sessions was entered into an excel spreadsheet, populated into a one to two-page document and forwarded to experts by the first author. Specialists in neurology, cardiology or geriatric medicine provided written feedback, commenting on existing risk management and providing recommendations for antithrombotic treatment. A hypothetical example of summaries mailed to GPs has been published [20] and is reproduced here (Fig. 1).

\section{Academic detailing only group}

GPs randomised to receive academic detailing only were mailed a summary of the proforma without expert specialist feedback. Proformas included a summary of the patient's risk of ischaemic stroke and safety messages 
70 year old [female/male] patient with atrial fibrillation [non-valvular] without thyrotoxicosis

SUMMARY OF PATIENT HISTORY

\begin{tabular}{|c|c|}
\hline Type of AF & Paroxysmal \\
\hline Ischaemic stroke risk factors & $\begin{array}{l}\text { Hypertension (controlled) } \\
\text { Diabetes }\end{array}$ \\
\hline CHADS $_{2}$ Score & 2 \\
\hline Annual Ischaemic Stroke Risk & $4.0 \%$ \\
\hline Other relevant co-morbidities & Nil reported \\
\hline Antithrombotic medications & Warfarin \\
\hline Other medications & Verapamil \\
\hline $\begin{array}{l}\text { Anticoagulant use-Current and } \\
\text { past }\end{array}$ & Yes \\
\hline $\begin{array}{l}\text { Adverse events/concerns } \\
\text { whilst on warfarin }\end{array}$ & $\begin{array}{l}\text { no major or minor bleeding and no ischaemic stroke } \\
\text { events }\end{array}$ \\
\hline $\begin{array}{l}\text { Reasons for not } \\
\text { prescribing warfarin }\end{array}$ & NA \\
\hline $\begin{array}{l}\text { Home Medicines Review (HMR) } \\
\text { considered? }\end{array}$ & No \\
\hline Webster packaging considered? & No \\
\hline $\begin{array}{l}\text { GP questions re } \\
\text { management/comments }\end{array}$ & $\begin{array}{l}\text { What is the role of aspirin in this patient, either used as a } \\
\text { single antithrombotic or in combination with warfarin? }\end{array}$ \\
\hline \multicolumn{2}{|c|}{$\begin{array}{l}\text { Specialist comments: Annual Ischaemic Stroke Risk: } 4.0 \% \\
\text { The risk of stroke is similar for both paroxysmal and chronic AF. Hence, a patient with a } \\
\text { CHADS } 2 \text { score of } 2 \text { should be on warfarin if they are in sinus rhythm but have a history of } \\
\text { paroxysmal AF. Aspirin reduces the relative risk of a stroke in AF by } 19 \% \text { over placebo and } \\
\text { hence does have a role - it is better than no antithrombotic therapy. However, warfarin has a } \\
\text { relative risk reduction over aspirin of } \sim 39 \% \text { and is more effective than aspirin in patients } \\
\text { with paroxysmal or chronic AF and a CHADS } 2 \text { score of } 2 \text { or higher. There is no evidence of } \\
\text { any additional benefit of combination aspirin and warfarin versus warfarin alone for AF. I } \\
\text { would also have concerns about an additive increased risk of bleeding with such a } \\
\text { combination. }\end{array}$} \\
\hline
\end{tabular}

Fig. 1 Expert summary delivered to GPs enrolled in the STOP-STROKE in AF study, 2010-2012. While this information is based on a real patient, demographic and disease characteristics have been modified to derive this hypothetical patient case. First published in Gattellari M, Worthington $J M$, Leung DY, Zwar. Supporting treatment decision making to optimise the prevention of stroke in atrial fibrillation: the STOP STROKE in AF study. Protocol for a cluster randomised controlled trial. Implementation Science, 2012; 7:63 https://doi.org/10.1186/1748-5908-7-63 Creative Commons Attribution License 4.0

were re-stated, where relevant (also included on the summary for GPs allocated to expert decisional support).

\section{Post-test data collection and outcomes}

Post-test assessment was scheduled within 12 weeks of the delivery of expert decisional support or patient summaries for GPs allocated to the academic detailing only arm.

The primary outcome was the proportion of cases recorded by GPs at post-test as currently receiving anticoagulant treatment, either with warfarin, clexane or dabigatran, the only DOAC first made available for managing AF in Australia from June 2011 through an industry funded familiarisation program. We selected anticoagulation as the primary outcome as barriers to the wider use of warfarin were predominately targeted given the evidence-based gap in managing AF related to concerns about anticoagulation and not antiplatelet use $[7,9,10]$. This outcome was also considered robust against reporting bias as treatment is readily ascertained by prescription records.

Secondary endpoints included antithrombotic treatment defined as appropriate if receiving 1) aspirin, 2) either an anticoagulant or antiplatelet, or 3) an anticoagulant if their baseline $\mathrm{CHADS}_{2}$ scores were 0,1 , or $2+$, respectively, according to evidence-based guidelines current at the time of the study [25]. As an additional secondary outcome, we qualified the above definition of "appropriate" antithrombotic treatment for 
patients receiving warfarin if their six most recent INR results indicated at least monthly assessment and at least four were within therapeutic range (ie, within 2.0-3.0) $[26,27]$. Patients younger than 65 years of age were excluded from the analysis of these secondary endpoints as pre-specified in our protocol [20] because the $\mathrm{CHADS}_{2}$ score was validated in patients aged over the age of 65 years.

"Appropriate" antithrombotic treatment was assessed using $\mathrm{CHADS}_{2}$ risk factors ascertained at baseline as the assessment and recording of these comorbidities during the course of the trial could have been influenced by the intervention itself potentially leading to differential ascertainment between groups. As we could not independently assess stroke risk factors at post-test, this outcome was designated a secondary outcome. Thus, $\mathrm{CHADS}_{2}$ comorbidities were recorded prior to randomisation to avoid biased ascertainment.

During the course of the study, validation of the $\mathrm{CHA}_{2} \mathrm{DS}_{2}$-VASc score [28] was published at which point we incorporated this information into the education program. To ensure consistency across all cases, however, we used the $\mathrm{CHADS}_{2}$ score for outcome ascertainment as per the original protocol.

Levels of decisional conflict about warfarin were measured using a five-item scale [29], adapted by specifying warfarin and/or its prescription within each statement. For each patient, GPs indicated their level of agreement to six statements to assess the extent to which GPs had reservations about prescribing warfarin for the patient. Summed item scores ranged from 6 to 30 with higher scores indicating a greater degree of uncertainty about whether or not to prescribe warfarin.

\section{Sample size estimate}

We required a total of 340 patients in total to detect a $15 \%$ difference in the primary outcome, assuming a 50\% baseline rate of anticoagulant prescribing [30]. This estimate was multiplied by a design effect of 1.06 , accounting for the clustered data, assuming an intraclass correlation coefficient of 0.029 [27], and, to produce an anticonservative estimate for the required number of GPs, an average of three patients per GP. We inflated the total sample size of 361 patients $(1.06 \times 340=361)$ by $20 \%$ to adjust for losses to follow-up, producing a final estimate of 452 patient cases (226 per group) and 152 GPs (76 per group).

\section{Statistical analysis}

Analyses were carried out by a biostatistician (co-author Andrew Hayen) blinded to group allocation. The analyses were intention-to-treat with patient data analysed according to group allocation. Log-binomial models were fitted to obtain relative risks for the analysis of dichotomous outcomes, while differences in decisional conflict scores were analysed using multiple linear regression. All analyses adjusted for the randomization stratum and accounted for clustering of patient data using generalised estimating equations. Criteria for statistical significance was a $p$-value of less than 0.05 . The significance of sub-group analyses was tested using interaction terms. Analyses were performed using SAS software (SAS institute Inc., Cary, USA).

\section{Results \\ Recruitment}

Of 5601 randomly selected GPs located around Australia, we contacted 5578 of whom 948 (17.0\%) expressed interest in receiving further information. Of these, 181 (19.1\% or $3 \%$ of all GPs approached) consented and completed the academic detailing session providing baseline data for 593 patients. Two GP trainees contributing three cases were excluded prior to randomization (Fig. 2), yielding a final baseline sample of 179 GPs and 590 cases. One-hundred and 18 GPs reported being in full-time practice $(65.9 \%$, including three missing responses), which is comparable with the national GP workforce in Australia during 2010/2011 ( $N=$ 17,951 out of 27,076 GPs or $66.3 \%$ in "full-time service"; http://www.health.gov.au/internet/main/publishing.nsf/ content/general+practice+statistics-1).

Eighty-nine GPs reporting 298 patient cases were randomly allocated to receive expert decisional support, while 90 GPs providing data on 292 cases received academic detailing only (Fig. 2). Of the 590 eligible patients, $48.0 \%$ were female $(N=283)$, while $59.0 \%(N=350)$ were aged 75 years or older. Half $(N=293,49.7 \%)$ were receiving anticoagulation at baseline.

Over-two thirds of patients $(N=401,68.0 \%)$ had a $\mathrm{CHADS}_{2}$ score of 2 or more. Only 51 (8.6\%) had a $\mathrm{CHADS}_{2}$ score of 0 . Baseline patient characteristics appeared balanced between groups (Table 1). Of the 179 enrolled GP, 152 completed 497 post-test audits. The audit post-test completion rate was $84.2 \%$ and did not significantly differ between groups $\left(\chi^{2}{ }_{(1)}=0.47, p=0.49\right)$ (Fig. 2).

There was evidence that cases lost to follow-up had a higher stroke risk and were more likely to be receiving anticoagulation at baseline. Of the 93 cases lost to follow-up (15.8\%), 58.1\% $(N=54)$ received anticoagulation at baseline, compared with $47.5 \%(N=236 / 497)$ of those with follow-up data, a near significant difference $\left(\chi^{2}{ }_{(1)}=3.51, p=0.06\right)$. Mean $\mathrm{CHADS}_{2}$ scores were lower in patients lost-to-follow-up (Mean $=2.1, \quad \mathrm{SD}=1.3)$, compared with those for whom follow-up data were obtained (Mean $=2.3, \mathrm{SD}=1.4$ ), although differences were not statistically significant $\left(t_{578}=1.75, p=0.08\right)$. There were no significant differences in sex, age group, median 


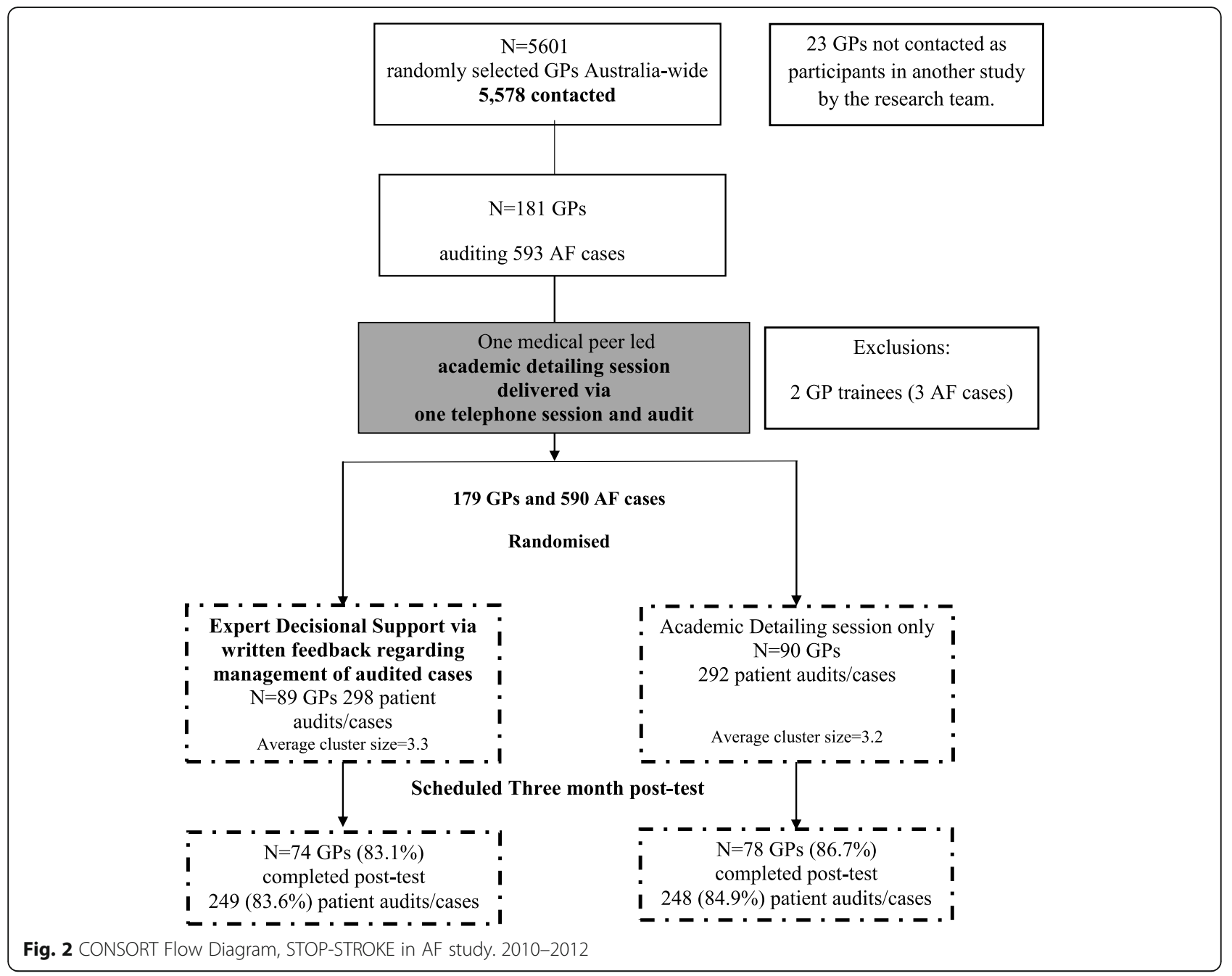

or mean age or median $\mathrm{CHADS}_{2}$ scores or $\mathrm{CHADS}_{2}$ categories between patients for whom post-audit data were and were not collected ( $\mathrm{p}$ 's $>0.10$ ).

\section{Primary outcome}

GPs receiving expert decisional support reported that $52.2 \%(N=130)$ of their patients were anticoagulated at post-test. By comparison, 47.6\% $(N=118)$ of patents were anticoagulated at post-test by GPs receiving academic detailing alone. This difference was not statistically significant (Adjusted Relative Risk $=1.11,95 \% \mathrm{CI}=$ 0.86 to 1.43$)(p=0.42)$ (Table 2$)$.

\section{Secondary outcomes}

Sixty-one percent of patients in both groups received appropriate antithrombotic management at post-test according to evidence-based guidelines (Adjusted $\mathrm{RR}=1.0$, $95 \% \mathrm{CI}=0.84$ to 1.18$)(p=0.97)$. Similarly, there was no difference between groups in the proportion of patients receiving appropriate antithrombotic management incorporating quality INR control criteria $(40.8 \%$ versus
45.0\%; Adjusted RR = 0.91, 95\% CI = 0.73-1.14, $p=0.43$ ). Planned sensitivity analyses for these secondary outcomes including patients younger than 65 years of age were not carried out given the null findings of the main analyses. Mean levels of decisional conflict were equivalent between groups $(p=0.44)$ (Table 2).

Responses to individual items comprising the decisional conflict scale are shown in Fig. 3. For around onein-five cases, GPs "strongly agreed" or "agreed" that they were unsure warfarin was the best treatment for the patient and whether the benefits of warfarin outweighed the risks or vice-versa. For one-third of selected cases, GPs "disagreed" or "strongly disagreed" that it was clear warfarin was the best treatment for the patient.

\section{Subgroup analyses}

Pre-specified subgroup analyses tested differences between groups on the primary outcome according to baseline $\mathrm{CHADS}_{2}$ scores $(0,1$, or $\geq 2)$, anticoagulation use at baseline (yes versus no) and patient sex and age ( $<65$ years, $65-74$ years, $75-84$ years, $\geq 85$ years). There 
Table 1 Baseline comparison of patient characteristics by group, Stop-Stroke in AF study 2010-2012

\begin{tabular}{|c|c|c|c|}
\hline Variable & $\begin{array}{l}\text { Academic Detailing+ } \\
\text { Expert Decisional Support n (\%) } \\
\mathbf{N}=298\end{array}$ & $\begin{array}{l}\text { Academic } \\
\text { Detailing } \mathrm{n}(\%) \\
\mathbf{N}=292\end{array}$ & $\begin{array}{l}\text { Total n (\%) } \\
\boldsymbol{N}=590\end{array}$ \\
\hline \multicolumn{4}{|l|}{ Sex } \\
\hline Male & $152(51.0)$ & $155(53.1)$ & $307(52.0)$ \\
\hline Female & $146(49.0)$ & $137(46.9)$ & $283(48.0)$ \\
\hline \multicolumn{4}{|l|}{ Age Group } \\
\hline$<65$ & $27(9.1)$ & $30(10.3)$ & $57(9.7)$ \\
\hline $65-74$ & $94(31.5)$ & $89(30.5)$ & $183(31.0)$ \\
\hline $75-84$ & $117(39.3)$ & $114(39.0)$ & $231(39.2)$ \\
\hline $85+$ & $60(20.1)$ & $59(20.2)$ & $119(20.2)$ \\
\hline Mean (SD) & $76.5(9.5)$ & $76.5(9.2)$ & $76.5(9.4)$ \\
\hline Median (IQR)S & $77.5(70-84)$ & $77.0(70-83)$ & $77(70-84)$ \\
\hline \multicolumn{4}{|l|}{$\mathrm{CHADS}_{2}$ score } \\
\hline 0 & $22(7.4)$ & $29(9.9)$ & $51(8.6)$ \\
\hline 1 & $57(19.1)$ & $71(24.3)$ & $128(21.7)$ \\
\hline $2+$ & $212(71.1)$ & $189(64.7)$ & $401(68.0)$ \\
\hline N/A (Cases with valvular AF) & $7(2.3)$ & $3(1.0)$ & $10(1.7)$ \\
\hline Mean (SD) & $2.4(1.4)$ & $2.2(1.4)$ & $2.3(1.4)$ \\
\hline Median (IQR) & $2(1-3)$ & $2(1-3)$ & $2(1-3)$ \\
\hline \multicolumn{4}{|l|}{ Baseline anticoagulation } \\
\hline Yes & $153(51.3)$ & $139(47.6)$ & $292(49.5)$ \\
\hline Previous Use & $46(15.4)$ & $47(16.1)$ & $93(15.8)$ \\
\hline No & $96(32.2)$ & $105(36.0)$ & $201(34.1)$ \\
\hline Missing & $3(1.0)$ & $1(0.3)$ & $4(0.7)$ \\
\hline \multicolumn{4}{|l|}{ Type of antithrombotic } \\
\hline Aspirin & $92(30.9)$ & $102(34.9)$ & $194(32.9)$ \\
\hline Clopidogrel & $12(4.0)$ & $13(4.5)$ & $25(4.2)$ \\
\hline Combined Antiplatelet $^{\mathrm{a}}$ & $12(4.0)$ & $17(5.8)$ & $29(4.9)$ \\
\hline Anticoagulant+Antiplatelet ${ }^{\mathrm{b}}$ & $13(4.4)$ & $17(5.8)$ & $30(5.1)$ \\
\hline Warfarin & $125(41.9)$ & $104(35.6)$ & $229(38.8)$ \\
\hline Dabigatran & $14(4.7)$ & $16(5.5)$ & $30(5.1)$ \\
\hline Clexane & $1(0.3)$ & $2(0.7)$ & $3(0.5)$ \\
\hline None & $29(9.7)$ & $21(7.2)$ & $50(8.5)$ \\
\hline
\end{tabular}

N/A Not applicable

$S D$ Standard Deviation

IQR Interquartile Range

ancludes aspirin plus dipyridamole

${ }^{b}$ Four patients were using dabigatran

was no evidence that the impact of group allocation on the primary outcome depended on patient age, sex, baseline $\mathrm{CHADS}_{2}$ score or baseline use of anticoagulation (p-values interaction $>0.05)$.

Amongst patients with available follow-up data, 214 out of 236 (90.7\%) receiving anticoagulation at baseline were still receiving it at post-test, while 11 out of 85 whose GPs reported at baseline their anticoagulation medication had been discontinued resumed treatment at post-test (12.9\%). Of the 176 patients with no history of anticoagulation use at baseline, $23(13.1 \%)$ received it at post-test.

\section{Discussion}

Main findings

Specialist feed-back in addition to a self-audit facilitated by peer-led academic detailing did not increase anticoagulant prescribing in Australian general practice. We 
Table 2 Comparison of primary and secondary outcomes reported at post-test, by group, Stop Stroke in AF study 2010-2012

\begin{tabular}{|c|c|c|c|c|c|}
\hline Variable & $\begin{array}{l}\text { Expert Decisional Support } \mathrm{n} / \mathrm{N} \\
(\%)\end{array}$ & $\begin{array}{l}\text { Academic Detailing alone } \mathrm{n} / \mathrm{N} \\
(\%)\end{array}$ & $\begin{array}{l}\text { Effect } \\
\text { Estimate }\end{array}$ & $95 \% \mathrm{Cl}$ & $\begin{array}{l}\boldsymbol{p}^{-} \\
\text {value }\end{array}$ \\
\hline \multicolumn{6}{|l|}{$\overline{\text { Primary outcome } e^{a}}$} \\
\hline Receiving anticoagulant therapy $(N=497)$ & $130 / 249(52.2)$ & 118/248 (47.6) & $\mathrm{RR}=1.11$ & $\begin{array}{l}0.86- \\
1.43\end{array}$ & 0.42 \\
\hline \multicolumn{6}{|l|}{ Secondary outcomes } \\
\hline Appropriate antithrombotic treatment $(N=443)^{b}$ & 136/223 (61.0) & $134 / 220(60.9)$ & $\mathrm{RR}=1.00$ & $\begin{array}{l}0.85- \\
1.19\end{array}$ & 0.97 \\
\hline $\begin{array}{l}\text { Appropriate antithrombotic incorporating quality control criteria } \\
(N=443)^{b}\end{array}$ & $91 / 223(40.8)$ & $99 / 220(45.0)$ & $R R=0.91$ & $\begin{array}{l}0.73- \\
1.14\end{array}$ & 0.43 \\
\hline Decisional Conflict $(N=497)$ & $\begin{array}{l}\text { Mean }=16 \\
95 \% \mathrm{Cl}=15.3-16.7\end{array}$ & $\begin{array}{l}\text { Mean }=16.6 \\
95 \% \mathrm{Cl}=16.0-17.2\end{array}$ & $\beta=-0.50$ & $\begin{array}{l}-1.79- \\
0.77\end{array}$ & 0.44 \\
\hline
\end{tabular}

Academic Detailing = Referent group

antraclass correlation coefficient for the primary outcome was 0.35

${ }^{b}$ Outcome was assessed only for patients aged 65 years or older as specified in protocol

addressed GPs' concerns about specific cases and detailed medical histories informed specialist feedback. This is in contrast to traditional methods for providing feedback using aggregated statistics and benchmark targets. Yet this novel method was not effective in promoting anticoagulant prescribing.

\section{Comparison with other studies}

Audit and feedback and academic detailing do not consistently promote evidence-based practice $[13,14]$ and our findings are in step with other evaluations of these strategies undertaken in a variety of contexts. Results are also consistent with findings from another study evaluating a multifaceted intervention for anticoagulation prescribing in AF, incorporating academic detailing and audit with feedback as we have done [16]. Moreover, three secondary stroke prevention trials did not report increased rates of anticoagulant prescribing for patients in $\mathrm{AF}$ in response to structured discharge care plans or nurse-led care [19, 31, 32].

Frequent feedback, using more than one delivery format, implementing action plans and providing targets enhance the effectiveness of audit and feedback [14]. While feedback here was delivered twice, verbally and in writing, we did not include action plans or specific targets and this may have underpinned the null findings. While feedback delivered by stroke specialists was hypothesised to be persuasive, the influence of experts may have been limited because they were not otherwise known to GPs nor involved in the care of their patients. This finding is consistent with results from another community-based study evaluating written, evidence-based summaries provided by opinion leaders, showing no improvement in the management of heart failure or ischaemic heart disease [33].

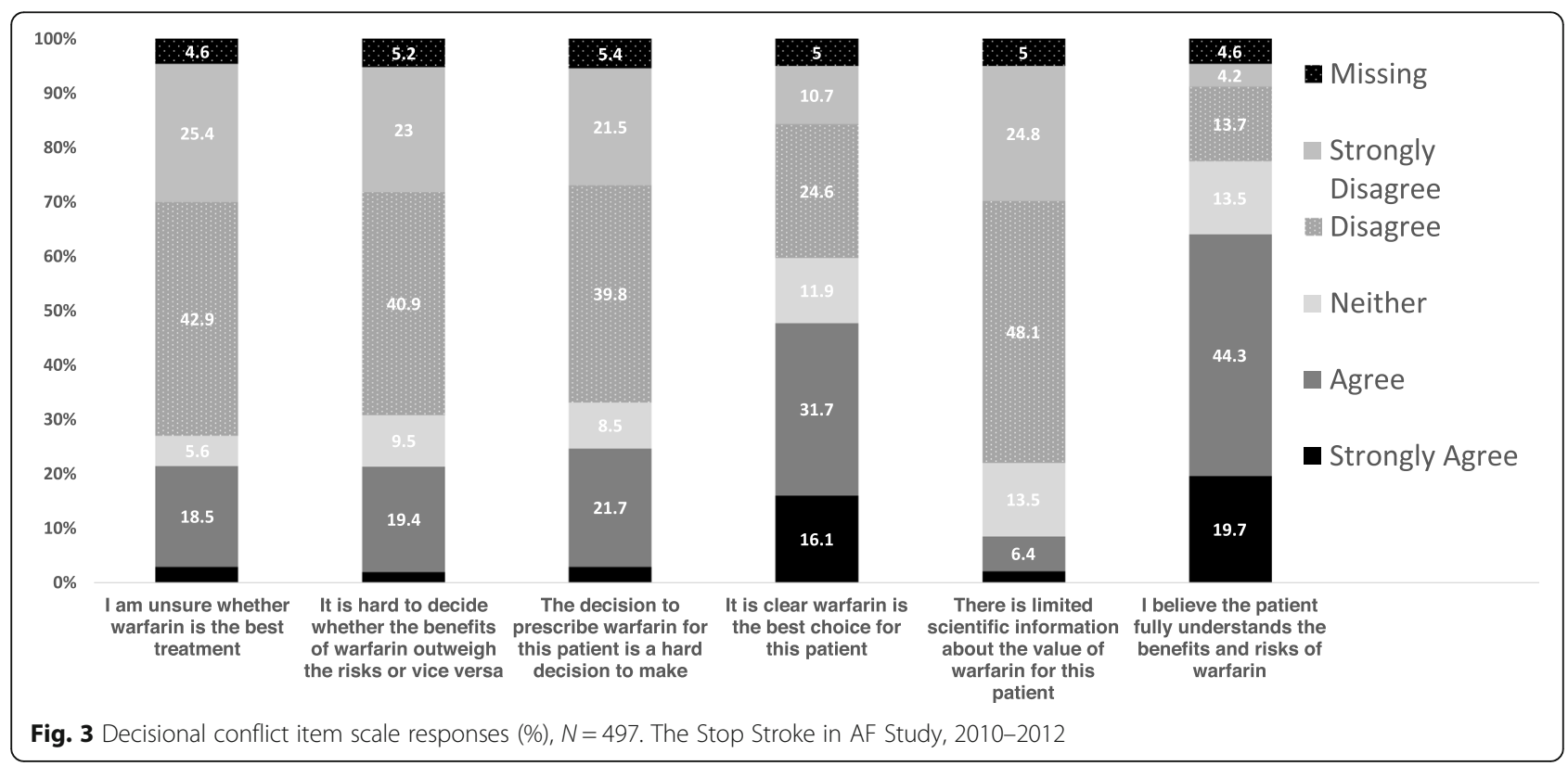


The intervention focussed on GPs while patients were not engaged. A recent study reported a small (3\%) but significant increase in anticoagulant prescribing attributable to a computerised decision support tool activated during interactions between physicians and patients [34], consistent with results from an earlier study employing a computerised risk assessment tool to guide real-time decision making [35]. In contrast, another recent study found no effect of an intervention using email notifications reporting patient-specific stroke risk and guideline recommendations for antithrombotic treatment [36]. Evidence from other randomised and non-randomised trials suggests simply having access to support tools, such as risk calculators, electronically generated feedback reports and automated prompts in anticipation of patient visits does not impact prescribing rates [37-39]. Taken together, findings from this and our own research imply that interventions delivered outside the context of the consultation are ineffective, yet providing opportunities for GPs to engage patients in discussions during real-time decision making may drive changes in prescribing behaviour.

The selection of patients by GPs in whom anticoagulation has been challenging may have made change especially difficult to achieve as there was no meaningful differences in anticoagulation rates from baseline to post-test in either group. The intervention therefore can be considered ineffective for patients selected in this way and the results may not generalise to all AF patients in general practice, for example, patients newly diagnosed with AF who are naïve to anticoagulants or those with less challenging needs. The impact of expert decisional support did not differ according to baseline patient characteristics, including baseline stroke risk. Therefore, there was no evidence that the impact of the intervention would have differed in patients with varying stroke risk. We acknowledge, however, that complex needs not reflected in the $\mathrm{CHADS}_{2}$ score, such as a patient's social and psychological circumstances, their cognitive capacity and adherence to medication may work against efforts to promote anticoagulation. While we did not systematically assess such barriers, these factors may have been relevant for the GPs. Moreover, there was no change in either group from baseline to post-test.

\section{Implications for current practice}

Our study was carried out as guidelines were evolving away from use of the $\mathrm{CHADS}_{2}$ score to the $\mathrm{CHA}_{2} \mathrm{DS}_{2}$ vasc score for risk stratification although US guidelines continued to recommend the use of $\mathrm{CHADS}_{2}$ sore in 2012 [40]. We had incorporated the $\mathrm{CHA}_{2} \mathrm{DS}_{2}$-vasc scheme to ensure GPs were aware of impending changes to local guidelines and feedback from experts also utilised this scheme where relevant. According to current recommendations [41], more AF patients are identified as suitable for anticoagulation, including those younger than 65 years of age. In this study, around $90 \%$ of patients were eligible for anticoagulation using the $\mathrm{CHADS}_{2}$ risk stratification scheme and therefore would have also been eligible under the $\mathrm{CHA}_{2} \mathrm{DS}_{2}$-vasc scheme. The main impact of the change in guidelines would have related to the 57 patients under 65 years of age in our study.

Antiplatelet medication has been largely superseded for the management of AF and recommendations now advise anticoagulation is to be preferred even in relatively low risk patients [42]. Real-world data indicates that a significant proportion $(\sim 15 \%)$ of patients with $\mathrm{AF}$ are prescribed antiplatelets, [43] and a recent study demonstrates no change in the proportion of patients prescribed aspirin since the change in guidelines [44], highlighting that current day treatment for AF remains sub-optimal for a sizeable proportion of patients.

The study was carried out before DOACs became widely available and at a time when their subsidised release under Australia's universal medical insurance scheme was imminent. Reluctance to prescribe warfarin may be intransigent. DOACs are perceived to be more convenient [45] with a proven lower risk of intracerebral haemorrhage [11] and these characteristics may leverage wider uptake of anticoagulation. Expert decisional support may have greater traction in the current postDOAC era as DOACs may be an "easier sell" if GPs are more pre-disposed to using these drugs over warfarin. However, our results are consistent with several randomised and non-randomised evaluations of implementation strategies for reducing stroke risk in AF which mainly report null findings or small differences in the uptake of anticoagulants in the pre and post-DOAC era [32, 36-39]. Taken together, these findings indicate that shifting prescribing practice remains challenging.

The recently published multicentre, multi-national IMPACT-AF study conducted in low to middle-income countries, incorporated both patient and GP education and closely monitored individual patients over a 12month period [46]. The study reported a $12 \%$ increase in anticoagulant prescribing over one-year compared with a $3 \%$ change in the usual care control arm. The results of IMPACT-AF may owe its success to persistent and sustained contact with both GPs and patients and the availability of DOACs. However, almost $80 \%$ of patients on anticoagulants were receiving warfarin at post-test, indicating a continued role for warfarin.

The study findings can be applied to current practice where warfarin is considered and continues to be used and, more generally, if the barriers to warfarin use also impede the uptake of DOACs. In Australia and elsewhere, DOAC dispensing has increased concomitantly 
with decreasing warfarin, although warfarin remains commonly prescribed [44, 47-55], and recent surveys show that warfarin is favoured amongst a significant proportion of clinicians [56], particularly for patients in whom use is established. In Australia, reports indicate that around one-quarter to one-third of initial anticoagulant prescribing is for warfarin $[48,49,54]$. There is also growing evidence that DOAC prescribing behaviour is influenced by the same perceived barriers that limited the wider use of warfarin indicating that DOACs do not appear to have bridged the evidence-practice gap as initially hoped and are subject to the same perceived limitations of warfarin. Recent studies report a high proportion of uncoagulated patients, for example, 32\% [43] and 57\% [52], and evidence also demonstrates that doctors remain cautious about bleeding risks, leading to non-evidence-based DOAC use. One large multicentre study of general practice patients showed that $40 \%$ of patients with $\mathrm{AF}$ and $40 \%$ of those at a high risk of stroke were not receiving anticoagulation despite trends towards increasing DOAC use [51]. Moreover, DOACs have been preferentially prescribed to younger patients, those with lower bleeding risks, fewer comorbidities and/or with lower stroke risk [50-53]. This has also been shown to occur in Australia, where patients with lower comorbidity burden are preferentially prescribed higher dose DOACs [57]. The underuse of anticoagulation in patients over the age of 85 remains significant [43]. In one analysis of over 100,000 anticoagulated AF patients, DOACs were associated with a higher risk of ischaemic stroke and shorter prothrombin time compared with warfarin use [58], suggesting under-coagulation in patients using these newer agents. Under-dosing is common [47, 59-62] and has been associated with an increased risk of thromboembolic events and mortality, although findings have not been consistent $[47,59-62]$. Contraindicated use or use inconsistent with recommendations is another concern [60, 61]. In an Australian study of AF patients admitted to hospital, 40\% were judged to have been prescribed a DOAC inappropriately. Specifically, DOACs were contraindicated in around one-in-eight patients, with both under-dosing and overdosing reported in patients $(\sim$ 15 and $10 \%$, respectively) [61].

Moreover, discontinuation of all oral anticoagulants is prevalent. In a study analysing data from over 45,000 newly-anticoagulated patients in the United States, 50\% receiving Apixaban, 65\% receiving Dabigatran, 57\% receiving Rivaroxaban and $71 \%$ receiving warfarin discontinued treatment within 1 year of initiating it and no more than $10 \%$ of patients switched to another agent [63]. In another large-scale study of over 64,000 patients, only $47 \%$ of those taking DOACs adhered to treatment for more than $80 \%$ of the time at a median of 1.1 years of follow-up, compared with $40 \%$ of those taking warfarin [64] while in another report based on over 62,000 patients newly diagnosed with AF, discontinuation of DOACs at 3 years was 46\% [51]. These results underscore that stroke risk reduction in AF remans ripe for knowledge translational activity and, contrary to expectations, barriers to wider and appropriate anticoagulation persist in contemporary practice.

DOACs were heralded to bridge the evidence-practice gap in AF management. Wider use of DOACs will increase the absolute numbers of patients who may experience a bleeding event if such patients would not have previously been anticoagulated given their risk of an adverse event would have been lower without any anticoagulation. If GPs encounter more patients experiencing adverse events, this may negatively reinforce their prescribing, resulting in more cautious future use and less evidence-based prescribing. These altering perceived risks may potentially impact on prescribing intentions, motivating GPs to reduce bleeding risk (or an act of commission) at the expense of reducing stroke risk (an act of omission) [12]. For these reasons, our results may inform future studies evaluating strategies to increase anticoagulation uptake.

\section{Strengths and limitations}

The large number of GPs involved in this study is a strength. Limitations include the unblinded self-reported outcomes and the $15 \%$ attrition rate of participants. Losses to follow-up were non-differential between groups and any bias would have likely favoured the intervention. Further, the three-month scheduled time between delivery of the intervention and post-test assessment may have limited the opportunities for GPs to change prescribing practices as recalling patients for a consultation was not specifically encouraged. Specialists to whom GPs referred patients may have been predominately responsible for determining treatment and GPs may have been reluctant to deviate from and contradict those decisions. The "control group" of the study was an active arm. Consequently, we do not know whether either expert decisional support, academic detailing or self-audit minimised discontinuation of anticoagulation compared to usual care. We do not have insights into GPs' views about the intervention as we did not conduct qualitative interviews at the end of the study. Specifically, it would be of interest to understand the reasons GPs did not change prescribing practice despite expert recommendations. We did not ask GPs whether they or another doctor had initiated treatment and do not know to what extent this contributed to the null findings. These issues are an important focus for future research.

GPs were advised to select patients for whom management presented challenges. This made case selection less 
costly in comparison to an independent audit. Only half of selected patient cases were receiving anticoagulation, even though $90 \%$ had a risk of stroke justifying anticoagulant use, characteristics which are consistent with those reported by others $[4,7,8]$. We saw evidence of decisional conflict with one-in-five GPs indicating difficulties in weighing up the benefits and risks of anticoagulation with one-third disagreeing that warfarin was the best choice for patients. These findings indicate that GPs selected appropriate cases for this knowledge translation exercise and self-audit may be relied upon in future research.

The selection of patients for whom management was considered challenging may have skewed the cohort towards a group of patients with more complex needs. More than two-thirds of patients had stroke risk warranting anticoagulation and $70 \%$ had a stroke risk assessed as "high" according to their $\mathrm{CHADS}_{2}$ score (that is, a score of two or more); fewer than $10 \%$ were deemed at low risk of stroke. The distribution of stroke risk is skewed towards higher risk compared with registrants in multicentre registries [65], although it is comparable to another knowledge translation Australian trial of AF patients recruited from general practice [35]. An audit of 82 Australian general practices carried out at the time this study was conducted reports just over onethird of patients having high risk of stroke compared with $70 \%$ here, although, like here, fewer than $10 \%$ were considered low risk [66].

The study cohort is best described as one of motivated volunteers as only $3 \%$ of GPs initially contacted eventually participated in the study. In the context of a randomised evaluation, however, comparisons should be internally valid. The national scope of the research and our limited resources precluded more resource intensive recruitment strategies, such as those involving follow-up phone calls, site visits and repeated mailings which would have likely increased participation. The characteristics of the selected cases are in keeping with previous studies $[4,7,8]$ and the proportion of GPs in full-time practice was consistent with national GP workforce statistics. Low participation rates amongst GPs in research studies is not an isolated experience. In an implementation trial carried out in the United Kingdom aimed at improving anticoagulant prescribing in AF, Holt et al. [37] recruited $8 \%$ of 570 general practices invited to participate via a mailed letter. Two Australian cluster randomised trials carried out in general practice have reported a $6 \%$ and an $8 \%$ participation rate amongst GPs located within the researchers' local areas which were predominately urban $[67,68]$. We utilised a study base of a random sample of GPs which could be expected to result in a lower participation rate compared with enrolling participants from a registry of self-nominated GPs interested in research.
To address the challenges in recruiting GPs, we deliberately approached a large number of potential participants and carried out two "Studies Within a Trial" (SWATs) evaluating various response-aiding strategies [20] at the time of initial contact. Interest in carrying out SWATs is gaining momentum to generate high quality evidence to improve trial administrative processes [69] and our findings will generate evidence to inform improved recruitment in primary health care implementation research.

\section{Conclusions}

Expert decisional support, delivered in writing, in addition to one academic detailing session administered via telephone by a medical peer compared with academic detailing alone, did not increase anticoagulant prescribing for patients GPs identified as not receiving anticoagulation or for whom management was perceived as challenging. Tested in this way, we found no support for its usefulness where warfarin was the predominately available anticoagulant. There may be merit in testing the effectiveness of specialist support using different methods of delivery (for example, a more interactive format), more frequent contact, focussing on newly diagnosed patients naïve to anticoagulation or in the context of increasing anticoagulant use [70], in which evidencepractice gaps persist $[47,50-53,59-62,71]$. On the basis of results reported here, expert decisional support cannot be recommended to improve anticoagulant prescribing in patients for whom anticoagulation is challenging.

\section{Abbreviations \\ AF: Atrial Fibrillation; ANZCTRN: Australian and New Zealand Clinical Trials Registration Number; DOAC: Direct Oral Anticoagulant; GP: General Practitioner}

\section{Acknowledgments}

We thank the GPs who participated and gratefully acknowledge the research assistance of Laura Morgan-Deller and Catherine Johnson which included recruiting GP participants and data collection. We thank Patrick McElduff, University of Newcastle for performing randomization. We extend our warmest gratitude to Drs Amelia Lem, Jemima Grant, Kate Johnston, Amelia Lem, Declan Mulvaney, Jay Ramanathan, Brian Simmons, and Anna Windsor for conducting academic detailing sessions. The STOP STROKE in AF specialist experts were Associate Professor David Blacker, Dr. Emma Blackwood, Professor Christopher Bladin, Associate Professor Brian Chambers, Associate Professor Dennis Cordato, Dr. Andy Evans, Associate Professor Stephen Read and Professor David Schultz. Co-authors Professor Dominic Leung and Associate Professor John M Worthington also provided expert input. Results of the study were presented as an oral presentation at the 25th Annual Scientific Meeting of the Stroke Society of Australasia. Gattellari M, Worthington JM, Leung DY, Hayen A, Zwar N. The STOP-STROKE in AF study. Supporting treatment decision making to optimise the prevention of stroke in atrial fibrillation. International Journal of Stroke 2015;10 (S3): 27.

\section{Authors' contributions}

Study concept and design: MG, JW, NZ, DL; Project management and supervision of study personnel: MG; Acquisition of data: MG; Data analysis: AH; Content expertise: JW, DL, NZ; Drafting of manuscript: MG; Interpretation of data and critical revision of manuscript: All authors. The authors read and approved the final manuscript. 


\section{Funding}

The STOP STROKE in AF trial was supported by the BUPA Foundation, Australia (formerly MBF Foundation). MG was supported by an NHMRC Public Health Post-Doctoral Training Fellowship (\#301136) and a Commonwealth Department of Health and Ageing, Primary Health Care Research, Evaluation and Development Mid-Level Career Research Fellowship during the conduct of this study. The opinions expressed in this publication do not necessarily reflect those of the Commonwealth of Australia, which does not accept any liability for loss, damage, or injury incurred by the use of or reliance on the information contained herein. The opinions expressed in this publication do not necessarily reflect those of the Commonwealth of Australia (which provided Fellowship support funding for the first author), which does not accept any liability for loss, damage, or injury incurred by the use of or reliance on the information contained herein.

\section{Availability of data and materials}

Data sharing was not a requirement at the time the study was conducted. Therefore, consent was not obtained from participants to share individual level data containing health information beyond the research team.

\section{Ethics approval and consent to participate}

The study was approved by The University of New South Wales Human Research Ethics Committee (\#07067). We obtained written consent from the study participants.

\section{Consent for publication}

Not applicable.

\section{Competing interests}

During 2017-2018, JMW and NZ were members of the National Heart Foundation of Australia and Cardiac Society of Australia and New Zealand Working Group (Honorary, unpaid positions) co-authoring the Australian Clinical Guidelines for the Diagnosis and Management of Atrial Fibrillation. JMW was an invited speaker by the Cardiac Society of Australia and New Zealand to present at the Society's 66th Annual Scientific Meeting in 2018 on guideline recommendations regarding secondary stroke prevention in atrial fibrillation (costs of travel were covered; speaker fees were not paid).

\section{Author details}

${ }^{1}$ Department of Neurology, Institute for Clinical Neurosciences, Neuroscience Research, Royal Prince Alfred Hospital, Missenden Road, Sydney Local Health District, Camperdown (Sydney), New South Wales 2050, Australia. ${ }^{2}$ Ingham Institute for Applied Medical Research, 1 Campbell Street, Liverpool, New South Wales 2170, Australia. ${ }^{3}$ Faculty of Health, University of Technology Sydney, 15 Broadway, Ultimo, New South Wales 2007, Australia. ${ }^{4}$ South Western Sydney Clinical School UNSW, Liverpool, Australia. ${ }^{5}$ Department of Cardiology, Liverpool Health Service, Sydney South West Local Health District, Clinical Services Building, Elizabeth Street, Liverpool (Sydney), New South Wales 2170, Australia. ${ }^{6}$ Faculty of Health, Sciences and Medicine, Bond University, 14 University Drive, Robina, Queensland 4226, Australia.

\section{Received: 11 March 2019 Accepted: 28 May 2020}

\section{Published online: 08 June 2020}

\section{References}

1. Feinberg WM, Blackshear $\mathrm{L}$, Laupacis $\mathrm{A}$, et al. Prevalence, age distribution and gender of patients with atrial fibrillation: analysis and implications. Arch Intern Med. 1995;155:469-73. https://doi.org/10.1001/archinte.1995. 00430050045005.

2. Wolf PA, Abbot RD, Kannel WB. Atrial fibrillation as an independent risk factor for stroke: the Framingham study. Stroke. 1991;22:983-8. https://doi. org/10.1161/01.STR.22.8.983.

3. Lin HJ, Wolf PA, Kelly-Hayes M, et al. Stroke severity in atrial fibrillation. The Framingham Study. Stroke. 1996;27:1760-4.

4. Steinberg BA, Greiner MA, Hammill BG, et al. Contradindications to anticoagulation therapy and eligibility for novel anticoagulants in older patients with atrial fibrillation. Cardiovasc Ther. 2015;33:177-83. https://doi. org/10.1111/1755-5922.12129.

5. Bassand J-P, Accetta G, Al Mahmeed W, et al. Risk factors for death, stroke, and bleeding in 28,628 patients from the GARFIELD-AF registry: rationale for comprehensive management of atrial fibrillation. PLoS One. 2018;13(1): e0191592. https://doi.org/10.1371/journal.pone.0191592.

6. Yu AYX, Malo S, Svenson LW, et al. Temporal trends in the use and comparative effectiveness of direct oral anticoagulant agents versus warfarin for nonvalvular atrial fibrillation: a Canadian population-based study. J Am Heart Assoc. 2017;6:e007129. https://doi.org/10.1161/JAHA.117. 007129

7. Ogilvie IS, Newton N, Welner SA, et al. Underuse of oral anticoagulants in atrial fibrillation: a systematic review. Am J Med. 2010;123:638-45. https:// doi.org/10.1016/j.amjmed.2009.11.025.

8. Xian Y, O'Brien EC, Liang $L, X u H$, et al. Association of preceding antithrombotic treatment with acute ischemic stroke severity and inhospital outcomes among patients with atrial fibrillation. JAMA. 2017; 317(10):1057-67. https://doi.org/10.1001/jama.2017.1371.

9. Katz DF, Maddox TM, Turakhia M, et al. Contemporary trends in oral anticoagulant prescription in atrial fibrillation patients at low to moderate risk of stroke after guideline-recommended change in Use of the $\mathrm{CHADS}_{2}$ to the $\mathrm{CHADS}_{2}$-vasc score for thrombotic risk assessment: Analysis from the National Cardiovascular Data Registry's Outpatient Practice Innovation and Clinical Excellence Atrial Fibrillation Registry. Circul Cardiovasc Qual Outcomes. 2017;10:e003476. https://doi.org/10.1161/CIRCOUTCOMES.116. 003476

10. Lubitz SA, Khurshid S, Weng C, et al. Predictors of oral anticoagulant non prescription in patients with atrial fibrillation and elevated stroke risk. Am Heart J. 2018;200:24-31. https://doi.org/10.1016/j.ahj.2018.03.00.

11. López-López José A, Sterne JAC, Thom HHZ, et al. Oral anticoagulants for prevention of stroke in atrial fibrillation: systematic review, network metaanalysis, and cost effectiveness analysis. BMJ. 2017;359. https://doi.org/10. 1136/bmj.j505.

12. Ezekowitz MD, Kent AP. The impact of IMPACT-AF. Lancet. 2017;390:1717-8. https://doi.org/10.1016/S0140-6736(17)32248-1.

13. O'Brien MA, Rogers S, Jamtvedt G, et al. Educational outreach visits: effects on professional practice and health care outcomes. Cochrane Database Syst Rev. 2007:4:CD000409. https://doi.org/10.1002/14651858.CD000409.pub2.

14. Ivers N, Jamtvedt G, Flottorp S, et al. Audit and feedback: effects on professional practice and healthcare outcomes. Cochrane Database Syst Rev. 2012;6:CD000259. https://doi.org/10.1002/14651858.CD000259.pub3.

15. Flodgren G, Parmelli E, Doumit G, et al. Local opinion leaders: effects on professional practice and health care outcomes. Cochrane Database Syst Rev. 2011;8:CD000125. https://doi.org/10.1002/14651858.CD000125.pub4.

16. Ornstein S, Jenkins RG, Nietert PJ, et al. A multimethod quality improvement intervention to improve preventive cardiovascular care: a cluster randomised trial. Ann Intern Med. 2004;141:523-32. https://doi.org/10.7326/ 0003-4819-141-7-200410050-00008.

17. Wright J, Bibby J, Eastham J, et al. Multifaceted implementation of stroke prevention guidelines in primary care: cluster-randomised evaluation of clinical and cost effectiveness. Qual Saf Health Care. 2007;16:51-9. https:// doi.org/10.1136/qshc.2006.019778.

18. Jackson SL, Peterson GM, Vial JH. A community-based educational intervention to improve antithrombotic drug use in atrial fibrillation. Ann Pharmacother. 2004;38:1794-9. https://doi.org/10.1345/aph.1E152.

19. Joubert J, Reid C, Barton D, et al. Integrated care improves risk-factor modification after stroke: initial results of the integrated Care for the Reduction of secondary stroke model. J Neurol Neurosurg Psychiatry. 2009; 80:279-84. https://doi.org/10.1136/jnnp.2008.148122.

20. Gattellari M, Worthington JM, Leung DY, et al. Supporting treatment decision making to optimise the prevention of stroke in atrial fibrillation: the STOP STROKE in AF study. Protocol for a cluster randomised controlled trial. Implement Sci. 2012;7:63. https://doi.org/10.1186/1748-5908-7-63.

21. Australian Institute of Health and Welfare. Medical workforce 2011. National health workforce series no. 3. Cat. No. HWL 49. Canberra: AlHW; 2013.

22. Britt H, Miller GC, Henderson J, et al. A decade of Australian general practice activity 2004-05 to 2013-14. General practice series no. 37. Sydney: Sydney University Press; 2014.

23. Gage BF, Waterman AD, Shannon W, et al. Validation of clinical classification schemes for predicting stroke: results from the National Registry of atrial fibrillation. JAMA. 2001;285:2864-70. https://doi.org/10.1001/jama.285.22. 2864.

24. Hart RG, Pearce LA, Aguilar MI. Meta-analysis: antithrombotic therapy to prevent stroke in patients who have nonvalvular atrial fibrillation. Ann Intern Med. 2007;146:857-67. 
25. Albers GW, Amarenco P, Easton JD, et al. American College of Chest Physicians: antithrombotic and thrombolytic therapy for ischemic stroke: American College of Chest Physicians Evidence-based Clinical Practice Guidelines (8th edition). Chest. 2008;13:630S-69S. https://doi.org/10.1378/ chest.11-2302.

26. Gallus AS, Baker Rl, Chong BH, On behalf of the Australasian Society of Thrombosis and Haemostatis: Position statement, et al. Consensus guidelines for warfarin therapy. Recommendations from the Australasian Society of Thrombosis and Haemostatis. Med J Aust. 2000;172:600-5.

27. McAlister FA, Man-Son-Hing M, Straus SE, for the Decision Aid in Atrial Fibrillation (DAAFI) Investigators, et al. Impact of a patient decision aid on care among patients with nonvalvular atrial fibrillation: a cluster randomised trial. CMAJ. 2005;173:496-501. https://doi.org/10.1503/cmaj.050091.

28. Lip GY, Nieuwlaat R, Pisters $R$, et al. Refining clinical risk stratification for predicting stroke and thromboembolism in atrial fibrillation using a novel risk factor-based approach: the euro heart survey on atrial fibrillation. Chest. 2010;137(2):263-72. https://doi.org/10.1378/chest.09-1584.

29. Dolan JG. Method for evaluating health care providers' decision making: the provider decision process assessment instrument. Med Decis Mak. 1991;19: 38-41. https://doi.org/10.1177/0272989X9901900105.

30. Dupont WD, Plummer WD. Power and sample size calculations: a review and computer program. Control Clin Trials. 1990;11:116-28. https://doi.org/ 10.1016/0197-2456(90)90005-M.

31. Olaiya MT, Kim J, Nelson MR, et al. Effectiveness of a shared team approach between nurses and doctors for improved risk factor management in survivors of stroke: a cluster randomised controlled trial. Eur J Neurol. 2017; 24:920-8. https://doi.org/10.1111/ene.13306.

32. Wolfe CDA, Redfern J, Rudd AG, et al. Cluster randomised controlled trial of a patient and general practitioner intervention to improve the management of multiple risk factors after stroke: stop stroke. Stroke. 2010;41:2470-6. https://doi.org/10.1161/STROKEAHA.110.588046.

33. Majumdar SR, Tsuyuki RT, McAlister FA. Impact of opinion leader-endorsed evidence summaries on the quality of prescribing for patients with cardiovascular disease: a randomised controlled trial. Am Heart J. 2007; 153(22):e1-8. https://doi.org/10.1016/j.ahj.2006.07.030.

34. Karlsson D, Nilsson S, Bang $M$, et al. A clinical decision support tool for improving adherence to guidelines on anticoagulant therapy in patients with atrial fibrillation at risk of stroke: a cluster randomised trial in a Swedish primary care setting (the GDS-AF study). PLoS Med. 2018;15:e1002528. https://doi.org/10.1371/journal.pmed.1002528.

35. Bajorek BV, Magin PJ, Hilmer SN, et al. Optimizing stroke prevention in patients with atrial fibrillation: a cluster randomised controlled trial of a computerized antithrombotic risk assessment tool in Australian general practice, 2012-2013. Prev Chronic Dis. 2016;13:E90. https://doi.org/10.5888/pcd13.160078.

36. Ashburner JM, Atlas SJ, Khurshid S, et al. Electronic physician notifications to improve guideline-based anticoagulation in atrial fibrillation: a randomised controlled trial. J Gen Intern Med. 2018. https://doi.org/10.1007/s1 1606-018-4612-6.

37. Holt TA, Dalton A, Marshall T, et al. Automated software system to promote anticoagulation and reduce stroke risk: cluster randomised controlled trial. Stroke. 2017;48:787-90. https://doi.org/10.1161/STROKEAHA.116.015468.

38. Arts DL, Abu-Hanna A, Medlock SK, et al. Effectiveness and usage of a decision support system to improve stroke prevention in general practice: a cluster randomised controlled trial. PLoS One. 2017;12:e0170974. https://doi. org/10.1371/journal.pone.0170974.

39. Kapoor A, Amroze A, Golden J, Crawford S, O'Day K, Elhag R, Nagy A, Lubitz SA, Saczynski JS, Mathew J, McManus DD. Support-AF: piloting a multifaceted, electronic medical record-based intervention to improve prescription of anticoagulation. J Am Heart Assoc. 2018;7:e009946. https:// doi.org/10.1161/JAHA.118.009946.

40. Lansberg MG, O'Donnell MJ, Khatri P, et al. Antithrombotic and thrombolytic therapy for ischemic stroke: antithrombotic therapy and prevention of thrombosis, $9^{\text {th }}$ ed: American College of Chest Physicians. Chest. 2012;141(2 Suppl):e601S-36S. https://doi.org/10.1378/chest.11-2302.

41. January CT, Wann LS, Calkins H, Chen LY, Cigarroa JE, et al. AHA/ACC/ HRS Focused opdate of the 2014 AHA/ACC/HRS guideline for the management of patients with atrial fibrillation. Circulation 2019;140: e125-e151.

42. Lip GYH, Pan X, Kamble S, Kawabata H, et al. Discontinuation risk comparison among 'real-world' newly anticoagulated atrial fibrillation patients: Apixaban, warfarin, dabigatran, or rivaroxaban. PLoS One. 2018;13: e0195950. https://doi.org/10.1371/journal.pone.0195950.
43. Lacoin L, Lumley M, Ridha E, et al. Evolving landscape of stroke prevention in atrial fibrillation within the UK between 2012 and 2016: a cross-sectional analysis study using CPRD. BMJ Open. 2017;7:e015363. https://doi.org/10. 1136/bmjopen-2016-015363.

44. Sur NB, Wang K, Di Tullio MR, et al. Disparities and temporal trends in the use of anticoagulation in patients with ischemic stroke and atrial fibrillation. Stroke. 2019;50:1452-9. https://doi.org/10.1161/STROKEAHA.118.023959.

45. Aronis KN, Hylek EM. Evidence gaps in the era of non-vitamin K Oral anticoagulants. J Am Heart Assoc. 2018;7:e007338. https://doi.org/10.1161/ JAHA.117.007338.

46. Vinereanu $D$, Lopes $R D$, Bahit MC, et al. A multifaceted intervention to improve treatment with oral anticoagulants in atrial fibrillation (IMPACT-AF): an international, cluster randomised trial. Lancet. 2017;390:1737-46. https:// doi.org/10.1016/S0140-6736(17)32165-7.

47. Vinogradova Y, Coupland C, Hill T, Cox JH. Risks and benefits of direct oral anticoagulants versus warfarin in a real world setting: cohort study in primary care. BMJ. 2018;362. https://doi.org/10.1136/bmj.k2505.

48. Pol D, Curtis C, Ramukumar S, Bittinger L. NOACs now mainstream for the use of anticoagulation in non-valvular atrial fibrillation in Australia. Heart Lung Circul. 2018. https://doi.org/10.1016/j.hlc.2018.03.010.

49. Morgan A, Joshy G, Schaffer A, et al. Rapid and substantial increases in anticoagulant use and expenditure in Australia following the introduction of new types of oral anticoagulants. PLoS One. 2018;13(12):e0208824. https://doi.org/10.1371/journal.pone.0208824.

50. Ashburner JM, Singer DE, Lubitz SA, et al. Changes in use of anticoagulation in patients with atrial fibrillation within primary care network associated with the introduction of direct oral anticoagulants. Am Coll Cardiol. 2017; 120:786-91. https://doi.org/10.1016/j.amjcard.2017.05.055.

51. Douros A, Renoux C, Coulombe J, Suissa S. Patterns of long-term use of non-vitamin K antagonist oral anticoagulants for non-valvular atrial fibrillation: Quebec observational study. Pharmacoepidemiol Drug Safety. 2017;26:1546-54. https://doi.org/10.1002/pds.4333.

52. Zhu J, Alexander GC, Nazarian S, Segal JB. Trends and variation in oral anticoagulant choice in patients with atrial fibrillation. 2010-2017. Pharmacotherapy. 2018;38:907-20. https://doi.org/10.1002/phar.2158.

53. Huiart L, Ferdynus $C$, Renoux C, et al. Trends in initiation of direct oral anticoagulant therapies for atrial fibrillation in a national population-based cross-sectional study in the French health insurance databases. BMJ Open. 2018;8:e018180. https://doi.org/10.1136/bmjopen-2017-018180.

54. Pratt NL, Ramsay EN, Caughey GE, Roughead EE. Uptake of novel oral anticoagulants in Australia. Med J Aust. 2016;204:104-5. https://doi.org/10. 5694/mja15.01000

55. Zhang Y, Mendéz SJ, Scott A. Factors affecting general practitioners' decisions to adopt new prescription drugs - cohort analyses using Australian longitudinal physician survey data. BMC Health Serv Res. 2019;19: 94. https://doi.org/10.1186/s12913-019-3889-4.

56. Wutzler A, Krogias C, Grau A, Veltkamp R, Heuschmann PU, Haeusler KG. Stroke prevention in patients with acute ischemic stroke and atrial fibrillation in Germany - a cross-sectional survey. BMC Neurol. 2019;19:25. https://doi.org/10.1186/s12883-019-1249-y.

57. Pratt NL, Ramsay E, Ellett LMK, et al. Comparative effectiveness and safety of low-strength and high-strength direct oral anticoagulants compared with warfarin: a sequential cohort study. BMJ Open. 2019;9:e0264886. https://doi. org/10.1136/bmjopen-2018-026486.

58. Shpak M, Ramakrishnan A, Nadasdy Z, Cowperthwaite M, Fanale C. Higher incidence of ischemic stroke in patients taking novel oral anticoagulants. Stroke. 2018;49:2851-6. https://doi.org/10.1161/ STROKEAHA.118.022636.

59. Sato T, Aizawa Y, Fuse K, Fujita S, Ikeda Y, Kitazawa H, Takahashi M, Okabe M. The comparison of inappropriate low dose use among 4 direct oral anticoagulants in patients with atrial fibrillation: from the database of a single centre registry. J Stroke Cerebrovasc Dis. 2018;27:3280-8. https://doi. org/10.1016/j.jstrokecerebrovasdis.2018.07.028.

60. Barra ME, Fanikos J, Connors JM, Sylvester KW, Piazza G, Goldhaber SZ. Evaluation of dose-reduced direct oral anticoagulant therapy. Am J Med. 2016;129:1198-204. https://doi.org/10.1016/j.amjmed.2016.05.041.

61. Pattullo CS, Barras M, Tai B, McKean M, Donovan P. New oral anticoagulants: appropriateness of prescribing in real-world setting. Intern Med J. 2016;46: 812-8. https://doi.org/10.1111/imj.13118.

62. Nielsen PB, Skjøth F, Søgaard M, et al. Effectiveness and safety of reduced dose non-vitamin $\mathrm{K}$ oral anticoagulants and warfarin in patients with atrial 
fibrillation: propensity weighted nationwide cohort study. BMJ. 2017;357: j510. https://doi.org/10.1136/bmj.j510.

63. Yao X, Abraham NS, Alexander GC, Crown W, Montori VM, Sangaralingham $L R$, Gersh BJ, Shah ND, Noseworthy PA. Effect of adherence to oral anticoagulants on risk of stroke and major bleeding among patients with atrial fibrillation. J Am Heart Assoc. 2016;5:e003074. https://doi.org/10.1161/ JAHA.115.003074.

64. Lip GYH, Banerjee A, Boriani G, et al. Antithrombotic therapy for atrial fibrillation. chest guideline and expert panel report. Chest. 2018;154:1121201. https://doi.org/10.1016/j.chest.2018.07.040.

65. Marcucci M, Lip GYH, Nieuwlaat R, et al. Stroke and bleeding risk codistribution: real world patients with atrial fibrillation. Am J Med. 2014;127: 979-86. https://doi.org/10.1016/j.amjmed.2014.05.003.

66. Family Medicine Research Centre. SAND Abstract No. 174 from the BEACH program 2010-11. Subject: Atrial fibrillation/flutter in general practice patients. The University of Sydney. https:/sydney.edu.au/medicine/fmrc/publications/ sand-abstracts/174-Atrial_fibrillation_flutter.pdf Accessed 10/10/2019.

67. Mills L, Meiser B, Ahmad R, et al. A cluster randomised controlled trial of an online psychoeducational intervention for people with a family history of depression. BMC Psychiatry. 2019;19:29. https://doi.org/10. 1186/s12888-018-1994-2.

68. Pond D, Mate K, Stocks N, et al. Effectiveness of a peer-mediated educational intervention in improving general practitioner diagnostic assessment and management of dementia: a cluster randomised controlled trial. BMJ Open. 2018;8:e21125. https://doi.org/10.1136/bmjopen-2017-021125.

69. Treweek S, Bevan S, Bower P, et al. Trial forge guidance 1: what is a study within a trial (SWAT). Trials. 2018;19:139. https://doi.org/10.1186/ s13063-018-2535-5.

70. Gadsbøll K, Staerk L, Fosbøl EL, et al. Increased use of oral anticoagulants in patients with atrial fibrillation: temporal trends from 2005 to 2015 in Denmark. Eur Heart J. 2017;38:899-906.

71. Steinberg BA, Shrader P, Pieper K, et al. Frequency and outcomes of reduced dose non-vitamin $\mathrm{K}$ antagonist anticoagulants: results from ORBITAF II (The outcomes registry for better informed treatment of atrial fibrillation II). J Am Heart Assoc. 2018;7:e007633. https://doi.org/10.1161/ JAHA.117.007633.

\section{Publisher's Note}

Springer Nature remains neutral with regard to jurisdictional claims in published maps and institutional affiliations.

Ready to submit your research? Choose BMC and benefit from:

- fast, convenient online submission

- thorough peer review by experienced researchers in your field

- rapid publication on acceptance

- support for research data, including large and complex data types

- gold Open Access which fosters wider collaboration and increased citations

- maximum visibility for your research: over $100 \mathrm{M}$ website views per year

At $\mathrm{BMC}$, research is always in progress.

Learn more biomedcentral.com/submissions 